\title{
Chaos, a problem for experiment
}

\author{
The study of chaotic behaviour has thrown up a new vocabulary, and some intriguing concepts. But \\ recognizing chaos experimentally may be more difficult than has been supposed.
}

If thinking the unthinkable has become respectable, surely defining the undefinable is a goal at which to strive? That, no doubt, is the spirit in which $\mathrm{Li}$ and Yorke embarked in 1975 on their already classical definition of chaos (Am. Math. Monthly $82,985 ; 1975)$. The concept of chaos has since then emerged essentially distinct from the most familiar physical manifestation of disorderliness, randomness as made manifest in brownian motion. The interest, and importance, of what is now called chaos is that it arises as a consequence of strictly deterministic processes. Everything is calculable, and thus in principle predictable, but our ways of comprehending chaos are poorly developed. So by what conceit can Gribogi, Ottand and Yorke now claim (Phys. Rev. Lett. 50, 935; 1983) to have identified a novel kind of chaos?

Nobody denies the underlying practical importance of chaos in dynamical systems. In calculations of the evolution of meteorological patterns in the Earth's atmosphere, for example, experience in the past few years has shown that prediction and reality diverge after 10 days or so, not so much because the underlying dynamical system is incalculable but because the pattern of evolution is extremely sensitive to the initial conditions, which are even now only poorly known. Chaos is also common in biology. Students of population dynamics, for example, have to their surprise now recognized that when the sizes of living populations fluctuate widely, even wildly, from one season to the next, the underlying explanation may again be some kind of chaotic behaviour.

Why is all this comprehended only with such difficulty? Part of the problem is that we are trapped by what Thomas S. Kuhn would no doubt call the paradign of newtonian calculus.

Take an inclined surface (not necessarily a plane) bounded by two edges at the "top" and "bottom" of the incline, and conduct the simple experiment in which billiard balls are allowed to roll from various points $A$ on the upper edge. The objective is to establish the relationship between the set of points $A$ along the upper edge and the set of points $B$ along the lower edge at which the billiard balls arrive. Ordinarily, if the inclined surface is reasonably smooth, to each $A$ there will be a distinct $B$, so that the experiment will establish a mapping of the set of points $A$ onto the set $B$, which is tantamount to saying that there is a functional relationship between them. Formally, $B=f(A)$, and it is possible to calculate $f$ from the known 0028-0836/83/180015-02\$01.00 shape of the surface and the laws of motion.

Our common expectation is that if a billiard ball starts not from $A$ but from the nearby point $A+\delta A$, it will finish at $B+\delta B$, near in some sense to $B$. Indeed, it is also natural to expect that $A$ and $\delta B$ will be related to each other in the sense that the ratio $\delta A / \delta B$ will be finite and that as $\delta A$ becomes smaller (or tends to zero), so too will $\delta B$ and in such a way that the ratio $\delta B / \delta A$ approaches some well defined limit, which is the differential coef ficient of the function $f$.

This, the common expectation, should however be amply falsified by another equally common experience - that of the old-fashioned pinball machine in which an inclined plane is studded with an array of metal obstacles. The mapping of $A$ onto $B$ is then by no means orderly. Patches of the upper edge may be mapped onto the lower edge in such a way that they are jumbled up and may even overlap (so that it is no longer possible unambiguously to infer $A$ from $B$ ). It may even be that $\delta B$ is large even when $\delta A$ is small, so that the function $f(A)$ will not be everywhere differentiable.

In the past eight years, much has been learned about the behaviour of chaotic systems by the study of simple mechanical and mathematical models. At the same time, there has emerged a novel and intriguing vocabulary. Bifurcation is widely used, not so much for the effect of a pinball pin on the motion of a rolling sphere down an inclined plane but for the common observation that changing the parameters specifying a potentially chaotic system will usually force it into one of two alternative conditions depending on the initial conditions. "Attractor" is another old word used in a new guise to refer to those states that frequently crop up even in a chaotic system. And then there are "strange attractor" states of the system, determined by certain sets of initial conditions, in which chaos supervenes. Whimsically, the sets of initial conditions are called "basins".

What Yorke and his associates have now done is to construct a series of mathematical models of a chaotic system which has unexpected properties. The simplest case is that in which the positions of points in the complex plane are defined by the pair of recurrence relations $\theta_{n+1}=2 \theta_{n} \bmod 2 \pi$ and $z_{n+1}=\lambda z_{n}+\cos \theta_{n}$ where $\lambda$ is a number between 1 and 2. (The instruction $\bmod 2 \pi$ simply implies that one chooses some initial value of the angle, doubles it and keeps only the part of it less than $2 \pi$.) The question, as with the observation of spheres in a pinball machine, is to know how the set of all possible initial conditions is related to the eventual outcome of the recursion, and a few simple properties of the system are clear. If, for example, the recursion is started off with an angle that is zero, and if the initial value of $z$ is say 1 , the value of $z_{n}$ will be $\lambda^{n}$, which diverges to infinity because $\lambda$ is itself greater than 1. Indeed, almost all sets of initial conditions end up with $z$ equal to plus or minus infinity, which are thus attractors for the system. The interesting question is to define the range of starting conditions from which some other outcome is possible.

Even with this simple model of chaos, some remarkable circumstances arise. First, the boundary of the basin is nowhere differentiable which, on the analogy of the pinball machine, implies that no $B+\delta B$ lies near $B$. Worse still, the length of the boundary, which is some kind of curve specifying $z$ as a function of angle and which would then be expected to have a length of the order of $2 \pi$, turns out to have an infinite length - a property diagnostic of a set of points with non-integral or "fractal" dimension. Yorke and his colleagues promise to show evidence that the fractal dimension is, indeed, $2-(\ln \lambda) /(\ln 2)$.

An elaboration of the simple recurrence relation leads to still more surprising consequences. With the same definition of the angles, but with $z_{n+1}$ defined as $\alpha z_{n}+z_{n}{ }^{2}+\beta \cos \theta_{n}$, the boundaries of the basins again have fractal dimensions. Because of the $z^{2}$ term, every divergent point now diverges to plus infinity. For suitable choices of the parameters, there is also a set of starting conditions for which true chaotic behaviour supervenes. By a suitable choice of the parameters, however, it is possible to banish altogether truly chaotic behaviour but Yorke and his associates now report that even then, the semblance of it persists. They have been able to describe the behaviour of these systems only by numerical methods, but they have found that even when chaos is not strictly speaking possible, as many as $10^{7}$ steps in the iteration of their formulae may be necessary before the influence of the attractor at infinity is able to take over.

The practical importance of this result is considerable, especially in the now absorbing question whether it is possible to distinguish experimentally between chaos and statistical randomness. For if quasichaos can persist for such long periods, telling the difference may in some circumstances be impractical.

(c) 1983 Macmillan Journals 1.1d 